\title{
Fazit und Ausblick
}

Die Ausarbeitung der Überschneidungen der Rechnungslegung und der Inflation ist besonders in Anbetracht der gestiegenen Inflationsraten weiterhin bedeutsam. Aufgrund der Preisniveaustabilität ist anzunehmen, dass der Konflikt in Zukunft weiter von Bedeutung sein wird.

Durch die Arbeit konnte ein Einblick in den schleichenden Prozess der Geldentwertung gegeben werden und ein Verständnis für die dahintersteckende Dynamik geschafft werden.

Im Zug der Internationalisierung sind weitere Angleichungen des Handelsrechts an die IFRS anzunehmen, sodass eine getreue Darstellung das Vorsichtsprinzip verdrängen könnte. Dadurch würde die Differenz zwischen einer nominalen und einer realen Darstellung einer Gesellschaft verkleinert werden.

Grundsätzlich ist die beste Darstellung für den Jahresabschlussadressaten zu erzeugen, sodass ein tatsächliches Bild der Gesellschaft erstellt wird. Zusammenfassend lässt sich festhalten, dass vor diesem Hintergrund empfohlen wird, dass Unternehmen Angaben zu stillen Effekten machen sollten. 